Pacific Journal of Mathematics

CARDINAL INEQUALITIES FOR TOPOLOGICAL SPACES 


\title{
CARDINAL INEQUALITIES FOR TOPOLOGICAL SPACES INVOLVING THE WEAK LINDELOF NUMBER
}

\author{
Murray Bell, John Ginsburg, and Grant Woods
}

\begin{abstract}
Let $w L(X), \chi(X), \psi(X), c(X)$, and $\partial(X)$ denote respectively the weak Lindelof number, character pseudocharacter, cellularity, and tightness of a Hausdorff topological space $X$. It is proved that if $X$ is a normal Hausdorff space then $|X| \leqq$ $2^{\chi(x) w L(X)}$. Examples are given of a nonregular Hausdorff space $Z$ such that $|Z|>2^{x(Z) w L(Z)}$ and a zero-dimensional Hausdorff space $Y$ such that $|Y|>2^{\psi(Y) \partial(Y) w L(Y)}$. Define $r \psi(X)=$ $\min \{\kappa$ : each closed subset of $X$ is the intersection of the closures of $\kappa$ of its neighborhoods\}. It is proved that $c(X) \leqq$ $r \psi(X) w L(X)$. Related open questions are posed.
\end{abstract}

1. Introduction. Let $X$ be a Hausdorff topological space. The weak Lindelof number of $X$, denoted $w L(X)$, is defined to be $\min \{\kappa$ : each open cover of $X$ has a subfamily of cardinality no greater than $\kappa$ whose union is a dense subspace of $X$ \}. If $w L(X)=\boldsymbol{\aleph}_{0}$ we say that $X$ is weakly Lindelof; see [9] and [10] for information concerning these spaces. It is immediate that $w L(X) \leqq L(X)$, where $L(X)$ is the Lindelof number of $X$ (definitions of this and other cardinal functions are given below). It is only slightly less trivial to see that $w L(X) \leqq c(X)$, where $c(X)$ denotes the cellularity of $X$. The theme of this paper is the study of situation in which $L(X)$ and/or $c(X)$ can be replaced, in inequalities involving cardinal functions on $X$, by $w L(X)$. We also consider in detail several illuminating counterexamples which place bounds on the situations in which such substitutions can be made.

Perhaps the most famous inequality involving cardinal functions is Arhangel'skii's theorem [2], which answered a fifty year old question of Alexandroff. It asserts that if $X$ is a Hausdorff space then $|X| \leqq 2^{x(X) L(X)}$, where $\chi(X)$ denotes the character of $X$. It has also been proved that if $X$ is Hausdorff then $|X| \leqq 2^{x(X) c(X)}$ (see [4]). One is led to conjecture that the common generalization of these theorems is true, namely that $|X| \leqq 2^{x(X) w L(X)}$. In Theorem 2.1 we prove that if $X$ is a normal Hausdorff space this is true; the proof is a modification of a technique used by Pol [6] to give an elegant proof of Arhangel'skii's theorem. We then demonstrate the need for some separation axioms in the hypotheses of this theorem by exhibiting two examples; first a nonregular Hausdorff space $Z$ for which $|Z|>2^{x(Z) w L(Z)}$, and second a zero-dimensional Hausdorff space $Y$ for which $|Y|>2^{\psi(Y) \partial(Y) w L(Y)}$ (here $\psi(Y)$ and $\partial(Y)$ denote respec- 
tively the pseudocharacter and the tightness of $Y$ ). This latter example is interesting because, as remarked in [6], Pol's proof of Arhangel'skii's theorem can be easily extended to show that if $X$ is Hausdorff then $|X| \leqq 2^{\psi(X) \partial(X) L(X)}$. The character of $Y$ is large, however, and it remains an open question whether $|X| \leqq 2^{x(X) w L(X)}$ for each regular Hausdorff space $X$. It is also unknown whether $|X| \leqq 2^{\psi(X) a(X) w L(X)}$ for each normal Hausdorff space $X$. The example $Y$ mentioned above serves in many ways as a "universal counterexample", and we examine its properties in some detail.

In Theorem 3.1 we prove that $c(X) \leqq w L(X) r \psi(X)$, where $r \psi(X)=\min \{\kappa$ : each closed subset of $X$ is the intersection of the closure of $\kappa$ of its neighborhoods\}. This provides another link between cellularity and weak Lindelof number. Previously considered examples are examined again in the light of this theorem. The paper ends with a list of open questions suggested by the preceding theorems and examples.

All hypothesized topological spaces will be assumed to be infinite Hausdorff spaces. If any further separation axioms are assumed in the hypotheses of a theorem, they will be stated explicitly. The set of natural numbers is denoted by $N$. The cardinality of a set $S$ is denoted by $|S|$. If $\mathscr{F}$ is a collection of sets, then $\cup\{F: F \in \mathscr{F}\}$ is denoted by $\cup \mathscr{F}$. If $\kappa$ is a cardinal number, $\kappa^{+}$will denote its cardinal successor. If $\alpha$ is an ordinal number, let $\alpha+1$ denote its ordinal successor. If $S$ is a set then $[S]^{<\kappa}$ denotes the set of subsets of $S$ of cardinality less than $\kappa$. In Example 2.2 we make use of Martin's axiom together with the negation of the continuum hypothesis. This set-theoretic assumption is denoted by $M A+\neg C H$; see [4] or [8] for a discussion of it. We shall use the notation and terminology for cardinal functions employed in [4]. For the convenience of the reader we repeat some of the definitions contained therein.

The Lindelof number of a space $X$, denoted $L(X)$, is $\min \{\kappa$ : each open cover of $X$ has a subcover of cardinality no greater than $\kappa\}$. The cellularity of $X$, denoted $c(X)$, is $\sup \{\kappa: X$ has a family of pairwise disjoint nonempty open sets of cardinality $\kappa\}$. If $c(X)=\mathbf{S}_{0}$ we say that $X$ satisfies the countable chain condition and abbreviate this by writing " $X$ has c.c.c.". The density character of $X$, denoted $d(X)$, is $\min \{\kappa: X$ has a dense subset of cardinality $\kappa\}$. Let $p \in X$. The character (respectively pseudocharacter) of $X$ at $p$, denoted $\chi(p, X)$ (respectively $\psi(p, X))$ is $\min \{\kappa: X$ has a neighborhood base at $p$ of cardinality $\kappa\}$ (respectively $\min \{\kappa:\{p\}$ is the intersection of $\kappa$ open subset of $X\}$ ). The character (respectively pseudocharacter) of $X$, denoted $\chi(X)$ (respectively $\psi(X))$, is $\sup \{\chi(p, X): p \in X\}$ (respectively $\sup \{\psi(p, X): p \in X\})$. The tightness of $X$ at $p$, denoted 
$\partial(p, X)$ is $\min \{\kappa: p \in \operatorname{cl} A$ implies there exists $B \cong A$ with $p \in \operatorname{cl} B$ and $|B| \leqq \kappa\}$. The tightness of $X$, denoted $\partial(X)$, is $\sup \{\partial(p, X): p \in X\}$.

2. Bounds on the size of a space in terms of its weak Lindelof number.

TheOREM 2.1. Let $X$ be a normal space. Then $|X| \leqq 2^{x(X) w L(X)}$.

Proof. Let $\kappa=\chi(X) w L(X)$. For each $p \in X$ find an open neighborhood base $\{G(p, \alpha): \alpha<\kappa\}$ at $p$. For each ordinal $\alpha<\kappa^{+}$ we will define by transfinite induction a subset $A(\alpha)$ of $X$ such that:

(i ) $\alpha_{1}<\alpha_{2}$ implies $A\left(\alpha_{1}\right) \subseteq A\left(\alpha_{2}\right)$.

(ii) $A(\alpha)$ is closed in $X$.

(iii) $|A(\alpha)| \leqq 2^{\kappa}$.

(iv) If $\mathscr{Q} \leqq\{G(p, \gamma): p \in A(\alpha)$ and $r<\kappa\}$ and $|\mathscr{Q}| \leqq \kappa$ and $X-$ $\operatorname{cl}[\cup \mathscr{U}] \neq \varnothing$ then $A(\alpha+1)-\operatorname{cl}[\cup \mathscr{Q}] \neq \varnothing$.

After having constructed $\left\{A(\alpha): \alpha<\kappa^{+}\right\}$as described above, we will show that $X=\cup\left\{A(\alpha): \alpha<\kappa^{+}\right\}$. By (iii) this will imply than $|X| \leqq 2^{\kappa} \cdot \kappa^{+}=2^{\kappa}$.

Let $S \subseteq X$ and let $p \in \operatorname{cl} S$. For each $\alpha<\kappa$ choose $x(\alpha) \in S \cap$ $G(p, \alpha)$ and put $T(p)=\{x(\alpha): \alpha<\kappa\}$. Define a function $\lambda: \operatorname{cl} S \rightarrow[S]^{\leqq \kappa}$ by $\lambda(p)=T(p)$. As $X$ is Hausdorff, $\lambda$ is one-to-one. Thus $|\operatorname{cl} S| \leqq|S|^{\kappa}$, so in particular if $|S| \leqq 2^{\kappa}$ then $|\mathrm{cl} S| \leqq 2^{\kappa}$.

Choose $x_{0} \in X$ and let $A(0)=\left\{x_{0}\right\}$. Now let $\alpha_{0}<\kappa^{+}$and suppose that we have chosen $\left\{A(\alpha): \alpha<\alpha_{0}\right\}$ so that (i)-(iv) are satisfied whenever the ordinal variables range over ordinals less that $\alpha_{0}$. We now define $A\left(\alpha_{0}\right)$. If $\alpha_{0}$ is a limit ordinal, let $A\left(\alpha_{0}\right)=\operatorname{cl}\left[\cup\left\{A(\alpha): \alpha<\alpha_{0}\right\}\right]$. Then (i)-(iv) are satisfied whenever the ordinal variables range over ordinals less than $\alpha_{0}+1$. Note that (iv) is satisfied vacuously when $\alpha=\alpha_{0}$ as $A\left(\alpha_{0}+1\right)$ is not defined. If $\alpha_{0}=\gamma_{0}+1$, let $\mathbb{}\left(\gamma_{0}\right)=$ $\left[\left\{G(p, \alpha): p \in A\left(\gamma_{0}\right) \text { and } \alpha<\kappa\right\}\right]^{\leqq \kappa}$. If $\mathscr{C} \in \mathbb{E}\left(\gamma_{0}\right)$ and $\operatorname{cl}[\cup \mathscr{C}] \neq X$ choose $r(\mathscr{C}) \in X-\operatorname{cl}\left[\cup \mathscr{C}\right.$ ]. Define $A\left(\alpha_{0}\right)=A\left(\gamma_{0}\right) \cup \operatorname{cl}\left\{r(\mathscr{C}): \mathscr{C} \in \mathbb{E}\left(\gamma_{0}\right)\right.$ and $X-\operatorname{cl}[\cup \mathscr{C}] \neq \varnothing\}$. Since $\left|A\left(\gamma_{0}\right)\right| \leqq 2^{\kappa}$ by hypothesis, it follows that $\left|\mathfrak{S}\left(\gamma_{0}\right)\right| \leqq\left(2^{\kappa}\right)^{\kappa}=2^{\kappa}$, so by the preceding paragraph $\left|A\left(\alpha_{0}\right)\right| \leqq 2^{\kappa}$. It follows immediately that (i), (ii), and (iv) follow whenever the ordinal variables range over values less than $\alpha_{0}+1$. The induction step is now complete.

Let $A\left(\kappa^{+}\right)=\bigcup\left\{A(\alpha): \alpha<\kappa^{+}\right\}$. Note that $A\left(\kappa^{+}\right)$is closed; for suppose $x \in \operatorname{cl} A\left(\kappa^{+}\right)$. For each $\alpha<\kappa$ choose $q(\alpha) \in A\left(\kappa^{+}\right) \cap G(x, \alpha)$. Then $q(\alpha) \in A\left(\mu_{\alpha}\right)$ for some $\mu_{\alpha}<\kappa^{+}$. Let $\mu=\sup \left\{\mu_{\alpha}: \alpha<\kappa\right\}$; then $\mu<\kappa$. Then $x \in \operatorname{cl}\{q(\alpha): \alpha<\kappa\} \cong \operatorname{cl} A(\mu) \subseteq A(\mu+1) \subseteq A\left(\kappa^{+}\right)$so $A\left(\kappa^{+}\right)$is closed. We claim that $A\left(\kappa^{+}\right)=X$; for if not, let $q \in X-A\left(\kappa^{+}\right)$. As $X$ is regular there exist disjoint open sets $U$ and $V$ with $q \in U$ and $A\left(\kappa^{+}\right) \subseteq V$. For each $p \in A\left(\kappa^{+}\right)$find $\alpha(p)<\kappa$ such that $G(p, \alpha(p)) \subseteq V$. 
Let $E=X-\cup\left\{G(p, \alpha(p)): p \in A\left(\kappa^{+}\right)\right\}$. Then $A\left(\kappa^{+}\right)$and $E$ are disjoint closed sets. As $X$ is normal, there exist disjoint closed sets $C$ and $D$ of $X$ such that $E \subseteq \operatorname{int} C$ and $A\left(\kappa^{+}\right) \subseteq \operatorname{int} D$. Then $X=(\operatorname{int} C) \cup$ $\left[\cup\left\{G(p, \alpha(p)): p \in A\left(\kappa^{+}\right)\right\}\right]$. As $w L(X) \leqq \kappa$ there exists a family $\left\{p_{i}: i<\kappa\right\}$ of points of $A\left(\kappa^{+}\right)$such that (int $\left.C\right) \cup\left[\cup\left\{G\left(p_{i}, \alpha\left(p_{i}\right)\right): i<\kappa\right\}\right]$ is dense in $X$. Now int $C \cap \operatorname{int} D=\varnothing$ so int $D \cap \cup\left\{G\left(p_{i}, \alpha\left(p_{i}\right)\right): i<\kappa\right\}$ is dense in int $D$. Thus

$$
A\left(\kappa^{+}\right) \subseteq \operatorname{cl}\left[\cup\left\{G\left(p_{\imath}, \alpha\left(p_{i}\right)\right): i<\kappa\right\}\right] \leqq \operatorname{cl} V \leqq X-U \subseteq X .
$$

If $\alpha_{0}=\sup \left\{\alpha\left(p_{i}\right): i<\kappa\right\}+1$, then $\alpha_{0}<\kappa^{+}$and $\mathscr{C}=\left\{G\left(p_{i}, \alpha\left(p_{i}\right)\right): i<\kappa\right\} \in$ $\mathfrak{C}\left(\alpha_{0}\right)$ by $(1)$. Hence $r(\mathscr{C}) \in A\left(\kappa^{+}\right)-\operatorname{cl}[\cup \mathscr{C}$ ], which contradicts (1). It follows that $A\left(\kappa^{+}\right)=X$ and so, as noted above, $|X| \leqq 2^{x(X) w L(X)}$.

One might be tempted to conjecture that each space $X$ (with sufficiently nice separation properties) has a dense subspace $S$ such that $L(S) \leqq w L(X)$ or $c(S) \leqq w L(X)$. If this were the case, then the inequality $|X| \leqq 2^{x(X) w L(X)}$ would follow from the three inequalities $|S| \leqq 2^{L(S) x(S)},|S| \leqq 2^{c(S) x(S)}$, and $|X| \leqq d(X)^{x(X)}$ (see [4] for proofs of there). To show that 2.1 does not follow from these inequalities in such a direct fashion, we give an example of a weakly Lindelof Tychonoff space all of whose Lindelof subspaces and c.c.c. subspaces are nowhere dense. We also exhibit a (consistent) example of a normal space with no dense Lindelof or c.c.c. subspace; however, we must use $M A+-C H$ to obtain this example.

ExAMPLE 2.2. Let $X$ be the Alexandroff double of the closed unit interval $I$ (see Example 2, page 107 of [3], or [1]). Thus, $X=I \times\{1,2\}$ topologized as follows: each point of $I \times\{1\}$ is isolated, while a neighborhood base at $(p, 2)$ is $\{[(p-1 / n, p+1 / n) \times\{2\}] \cup$ $[((p-(1 / n), p+(1 / n))-\{p\}) \times\{1\}]: n \in N\}$ for each $p \in I$. Then $X$ is a conpact Hausdorff first countable space with an uncountable collection of isolated points. Thus $X^{\omega}$ is compact Hausdorff and first countable, while each subset of $X^{\omega}$ of countable cellularity has an empty interior. Let $P$ be the space of finite subsets of $I$ with the Pixley-Roy topology. Thus if $A \in P$ and $U$ is open in $I$ with $A \subseteq U$, denote by $[A, U]$ the set $\{F \in P: A \subseteq F \leqq U\}$. Then $\{[A, U]: A \in P$, $U$ open in $I, A \subseteq U\}$ is a base for topology on $P$, the "Pixley-Roy topology". Pixley-Roy topologies are discussed at some length in [7], and the properties of $P$ mentioned below without explicit citations may be found therein. $P$ is first countable, has c.c.c. (and hence is weakly Lindelof), and each Lindelof subspace of $P$ is nowhere dense. Thus $X^{\omega} \times P$ is first countable and weakly Lindelof (being the product of a compact space and a weakly Lindelof space). If $S$ is a 
subspace of $X^{\omega} \times P$ that is either Lindelof or c.c.c. then $S$ is nowhere dense. Unfortunately $X^{\omega} \times P$ is not normal.

Now assume $M A+\neg C H$, and let $T$ be an uncountable subset of $I$ of cardinality less than $2^{\aleph_{0}}$. The space $P_{1}$ of finite subset of $T$, with the Pixley-Roy topology, enjoys all the properties of $P$ listed above except that $P_{1}$ is normal (see [7]). Thus the free union $X^{\omega} \cup P_{1}$ is first countable, normal, weakly Lindelof space with no dense Lindelof subspace and no dense subspace of countable cellularity.

Next we consider two examples which give bounds to the degree to which 2.1 can be generalized. First, we produce a Hausdorff space $Z$ for which $|Z|>2^{x(Z, w L(Z)}$. Second, we exhibit a zero-dimensional Hausdorff space $Y$ such that $|Y|>2^{\psi(Y) \partial(Y) w L(Y)}$. In fact the constructions of $Y$ and $Z$ are almost identical. We consider $Z$ first.

EXAMPLe 2.3. Let $\kappa$ be any uncountable cardinal, let $Q$ denote the rational numbers, and let $A$ be any countable dense subset of the space of irrational numbers. Then $Z$ is the set $(Q \times \kappa) \cup A$ topologized as follows. If $q \in Q$ and $\alpha<\kappa$ then a neighborhood base at $(q, \alpha)$ is $\left\{U_{n}(q, \alpha): n=1,2, \cdots\right\}$ where $U_{n}(q, \alpha)=\{(r, \alpha): r \in Q$ and $|r-q|<1 / n\}$. If $a \in A$, a neighborhood base at $a$ is $\{\{b \in A:|b-a|<$ $1 / n\} \cup\{(q, \alpha): \alpha<\kappa$ and $|q-a|<1 / n\}: n=1,2, \cdots\}$. It is easily seen that this assignment of neighborhood bases makes $Z$ into a first countable Hausdorff space in which, for each $\alpha<\kappa, Q \times\{\alpha\}$ is open in $Z$ and homeomorphic to $Q$ (with the usual topology). Now suppose that $G$ is an open subset of $Z$ containing $A$. We show that $G$ is dense in $Z$; i.e., we show that if $q \in Q, \alpha<\kappa$, and $n \in N$ are given, then $U_{n}(q, \alpha) \cap G \neq \varnothing$. To do this, note that as $A$ is dense in $R$ there exists $a \in A$ such that $|a-q|<1 / n$. As $G$ is open and contains $a$, there exists $m \in N$ such that $(r, \alpha) \in G$ whenever $r \in Q$ and $|r-a|<1 / m$. Choose $j \in N$ such that $j>m$ and $1 / j<$ $1 / n-|a-q|$. If $|r-a|<1 / j$ and $r \in Q$ then $(r, \alpha) \in G \cap U_{n}(q, \alpha)$. Hence $G$ is dense in $Z$. This implies that $Z$ is not regular. It also implies that $Z$ is weakly Lindelof, for any open cover of $Z$ contains a countable subfamily covering $A$ (as $A$ is countable) and the union of this countable family is dense in $Z$. Thus $\chi(Z)=w L(Z)=\boldsymbol{\aleph}_{0}$ and $|Z|=\kappa$. Hence the gap between $|Z|$ and $2^{x(Z) w L(Z)}$ is arbitrarily large.

EXAMPLe 2.4. Let $Y$ be identical to the space $Z$ of 2.3 except that the neighborhood bases at points of $A$ are changed as follows: if $a \in A, n \in N$, and $F \in[\kappa]^{<\omega}$, let $V_{n, F}(a)=\{\{b \in A:|b-a|<1 / n\} \cup$ $\{(q, \alpha) \in Q \times \kappa:|q-\alpha|<1 / n$ and $\alpha \notin F\}\}$. Then $\left\{V_{n, F}(\alpha): n \in N\right.$ and 
$\left.F \in[\kappa]^{<\omega}\right\}$ is taken as a neighborhood base at $a$. It is easily seen that this is a valid assignment of neighborhood bases at points and that $Y$ is a zero-dimensional Hausdorff space (and thus completely regular).

Now we show that is $G$ if an open set containing $A$, then $Y-\operatorname{cl} G$ is countable. This will immediately imply (as in 2.2 above) that $Y$ is weakly Lindelof, i.e., that $w L(Y)=\aleph_{0}$. Suppose that $Y-c l G$ is uncountable. Since $Q$ is countable there is $q \in Q$ and a subset $S$ of $\kappa$ such that $|S|=\aleph_{1}$ and $\{q\} \times S \subseteq Y-c l G$. For each $\alpha \in S$ there exists $n(\alpha) \in N$ such that $U_{n(\alpha)}(q, \alpha) \cap G=\varnothing$. By the pigeonhole principle there is a subset $T$ of $S$ and $n \in N$ such that $|T|=\aleph_{1}$ and $U_{n}(q, \alpha) \cap$ $G \neq \varnothing$ for each $\alpha \in T$. Since is dense $A$ in $R$ there exists $a \in A$ with $|a-q|<1 / n$. Since each basic neighborhood of $a$ is disjoint from at most finitely many sets of the form $Q \times\{\alpha\}$, where $\alpha<\kappa$, it easily follows (as in 2.2) that $a \in \operatorname{cl}\left[\cup\left\{U_{n}(q, \alpha): \alpha \in T\right\}\right]$. But $G$ is an open set containing $a$ and disjoint from $\cup\left\{U_{n}(q, \alpha): \alpha \in T\right\}$, which is a contradiction. Hence $Y-\operatorname{cl} G$ is countable as claimed.

We next show that $\partial(Y)=\boldsymbol{\aleph}_{0}$. Obviously $\partial(p, Y)=\aleph_{0}$ if $p \in$ $Q \times \kappa$ since basic neighborhoods of such points are countable. Let $a \in A, S \leqq Y$, and suppose $a \in \operatorname{cl} S$. We show that $S$ has a countable subset $S_{1}$ such that $a \in \operatorname{cl} S_{1}$. If $a \in \operatorname{cl}(S \cap A)$ then $S \cap A$ is our $S_{1}$. If not, then $a \in \operatorname{cl}(S \cap(Q \times \kappa))$. Inductively choose a sequence $S_{1}=$ $\left\{\left(q_{n}, \alpha_{n}\right): n \in N\right\}$ of points from $S \cap(Q \times \kappa)$ such that $\left|a-q_{n}\right|<1 / n$ and such that $n \neq m$ implies $\alpha_{n} \neq \alpha_{m}$; this is easily done using the fact that $V_{n, F}(a)$ meets $S \cap(Q \times \kappa)$ where $F=\left\{\alpha_{i}: i \leqq n-1\right\}$. Obviously $\left|S_{1}\right|=\boldsymbol{\aleph}_{0}$ and as each neighborhood of $a$ is disjoint from $Q \times\{\alpha\}$ for at most finitely many $\alpha$, it is easily seen that $a \in c l S_{1}$. Thus $\partial(a, Y)=\boldsymbol{\aleph}_{0}$ so $\partial(Y)=\boldsymbol{\aleph}_{0}$.

Next we show that every subset of $Y$ is a $G_{\delta}$-set of $Y$. Thus in particular $Y$ is perfect (i.e., each closed subset is a $G_{\delta}$-set) and $\psi(Y)=\boldsymbol{\gamma}_{0}$. To see this, note that for each $q \in Q,\{q\} \times \kappa$ is a closed discrete subset of $Y$. Thus $Y=\cup\{\{q\} \times \kappa: q \in Q\} \cup\{\{a\}: a \in A\}$ expresses $Y$ as a union of countably many closed discrete subspaces. Rewrite this as $Y=\cup\left\{D_{n}: n \in N\right\}$ where each $D_{n}$ is closed in $Y$ and discrete. If $S \subseteq Y$ then $S=\cup\left\{S \cap D_{n}: n \in N\right\}$ and each $S \cap D_{n}$ is closed in $Y$. Thus $S$ is an $F_{\sigma}$, and our claim follows.

Since $\kappa$ was arbitrary and $|Y|=\kappa$, we see that $|Y|=\kappa>2^{\aleph_{0}}=$ $2^{w L(Y) \psi(Y) \partial(Y)}$ if $\kappa>2^{\aleph_{0}}$. Hence we cannot simultaneously replace $\chi(X)$. by $\psi(X) \partial(X)$, and normality by complete regularity, in 2.1 .

The space $Y$ and its countable power $Y^{\omega}$ have a number of other interesting properties which we consider now. Obviously $\chi(a, Y)=\kappa$ for each $a \in A$, even though $\psi(a, Y)=\partial(a, Y)=\boldsymbol{\aleph}_{0}$. Also, if $\mathscr{G}$ is a family of open sets, each containing $A$, such that 
$A=\cap\{\mathrm{cl} G: G \in \mathscr{G}\}$, then $|\mathscr{G}| \geqq \kappa$, even though $A$ is a $G_{\tilde{r}^{-}}$set of $Y$. This illustrates that $Y$ is not normal. Also, it is easily seen that if $D$ is a dense subspace of $Y$ then $L(D)=c(D)=\kappa$. However, the set of points of $Y$ that have Lindelof neighborhoods, and the set of points of $Y$ that have c.c.c. neighborhoods, is just $Q \times \kappa$, which is dense in $Y$.

We next consider the countable power $Y^{\omega}$. We claim that it is a weakly Lindelof, perfect space of countable tightness in which each Lindelof subspace, and each subspace of countable cellularity, is nowhere dense. The last claim follows immediately from the fact that $Y$ is neither Lindelof nor of countable cellularity.

We sketch the argument that $Y^{\omega}$ is weakly Lindelof. First, by an argument similar to that used above in showing that $Y$ is weakly Lindelof, one observes that if $G$ is open in $Y^{2}$ and contains $A \times A$ then at most countably many points both of whose co-ordinates are rational lie outside $\mathrm{cl} G$. Thus, given an open cover of $Y$, we may find a countable subfamily $\mathscr{C}$ whose union covers the countable set $A \times A$; then $Y^{2}-\mathrm{cl}[\cup \mathscr{C}]$ contains only countably many points of the form $((q, \alpha),(r, \beta))$. The rest of $Y^{2}$ is contained in $\cup\{\{a\} \times Y: a \times A\} \cup$ $\cup\{Y \times\{a\}: a \in A\}$, a countable union of weakly Lindelof spaces. Thus we readily obtain a countable subfamily of the original open cover whose union is dense. This shows that $Y^{2}$ is weakly Lindelof. This argument easily extends by induction to show that each finite power $Y^{n}$ is weakly Lindelof. By Ulmer's theorem (1.3 of [9]) it follows that $Y^{\omega}$ is weakly Lindelof.

Recall $Y$ is a countable union of closed discrete subspaces; it is readily seen that this implies that each finite power $Y^{n}$ enjoys the same property. Thus each subset of $Y^{n}$ is a $G_{\tilde{o}}$-set of $Y^{n}$, so in particular each $Y^{n}$ is perfect. By Katetov's theorem (see [5]) it follows that $Y^{\omega}$ is perfect.

The argument given above to show that $\partial(Y)=\boldsymbol{\aleph}_{0}$ easily extends to show that $\partial\left(Y^{n}\right)=\boldsymbol{\aleph}_{0}$ for each $n \in N$. This clearly implies that $\partial\left(Y^{\omega}\right)=\aleph_{0}$.

3. A relationship between cellularity and weak Lindelof number. The examples in $2.2,2.3$, and 2.4 serve to illustrate that the weak Lindelof number is independent of both the Lindelof number and the cellularity, even for spaces with "nice" separation properties and for which the tightness and pseudocharacter are small. These examples also show that the class of weakly Lindelof spaces is much broader than the class of spaces with dense Lindelof subspaces, or dense subspaces of countable cellularity. Thus, based on what we have seen to date, we know that although $w L(X) \leqq c(X)$ and $w L(X) \leqq L(X)$, the gap between $w L(X)$ on one hand, and $c(X)$ 
and $L(X)$ on the other hand, can be arbitrarily large. We now give a condition under which the cellularity is bounded by the weak Lindelof number. Recall that the definition $r \psi(X)$ is given in $\S 1$.

THEOREM 3.1. $c(X) \leqq w L(X) r \psi(X)$.

Proof. Let $\kappa=w L(X) r \psi(X)$. Suppose $c(X) \geqq \kappa^{+}$; let $\mathscr{G}=$ $\left\{G_{i}: i<\kappa^{+}\right\}$be a family of $\kappa^{+}$pairwise disjoint nonempty open subsets of $X$. Let $F=X-\cup\left\{G_{i}: i<\kappa^{+}\right\}$. Then $F$ is closed in $X$. If $U$ is any open subset of $X$ containing $F$, then as $w L(X) \leqq \kappa$ there is subfamily $\mathscr{F}$ of $\mathscr{G}$ of cardinality no greater than $\kappa$ such that $(\cup \mathscr{F}) \cup U$ is dense in $X$. It follows that $\operatorname{cl} U$ contains all but $\kappa$ members of $\mathscr{G}$. As $r \psi(X) \leqq \kappa$, there is a family $\mathscr{U}$ of open sets containing $F$ such that $|\mathscr{U}| \leqq \kappa$ and $\cap\{\operatorname{cl} U: U \in \mathscr{C}\}=F$. For each $U \in \mathscr{Q}$ let $A(U)=\left\{i<\kappa^{+}: G_{i} \nsubseteq \mathrm{cl} U\right\}$. By the above $|A(U)| \leqq \kappa$, and so $|\cup\{A(U): U \in \mathscr{C}\}| \leqq \kappa$. Choose $j \in \kappa^{+}-\cup\{A(U): U \in \mathscr{Q}\}$. Then $G_{j} \leqq \cap\{\mathrm{cl} U: U \in \mathscr{U}\}=F$, which contradicts the definition of $F$. Thus $c(X) \leqq w L(X) r \psi(X)$ as claimed.

CoRollary 3.2. Let $\bar{\psi}(X)=\min \{\kappa$ : each closed subset of $X$ is the intersection of no more than $\kappa$ open sets $\}$. If $X$ is normal then $c(X) \leqq w L(X) \bar{\psi}(X)$. In particular every perfectly normal weakly Lindelof space has countable cellularity.

Proof. In a normal space $r \psi(X)=\bar{\psi}(X)$.

REMARK 3.3. Although it is obvious that $\bar{\psi}(X) \leqq r \psi(X)$ in general, the space $Y$ of Example 2.4 shows that the gap between $\bar{\psi}(X)$ and $r \psi(X)$ can be arbitrarily large; $\bar{\psi}(Y)=\boldsymbol{\psi}_{0}$ and $r \psi(Y)=\kappa$. If we did not know it already, we could deduce from 3.2 that $Y$ is nonnormal since $c(Y)=\kappa$ and $w L(Y)=\boldsymbol{\aleph}_{0}$.

\section{Open questions.}

4.1. Is either of the obvious generalization of 2.1 true? In other words, is $|X| \leqq 2^{\psi(X) \partial(X) w L(X)}$ if $X$ is normal, and is $|X| \leqq 2^{x(X) w L(X)}$ if $X$ is regular? Example 2.4 illustrates that we cannot make both generalizations simultaneously.

4.2. In Example 2.2 we used $M A+\neg C H$ to construct a normal, first countable, c.c.c. space with no dense Lindelof subspace. Is there a "real" example of such a space? Does such a "real" example exist if we replace "c.c.c." by "weakly Lindelof" in the above list of properties? 
4.3. Is there a theorem that relates $L(X)$ to $w L(X)$ in a manner analogous to the way in which 3.1 relates $c(X)$ to $w L(X)$ ? Aside from the observation that $L(X) \leqq w L(X) p(X)$ (where $p(X)$ is the "paracompactness number"; i.e., $p(X)=\min \{\kappa$ : each open cover of $X$ has an open locally $\kappa$ refinement\}), we know of no interesting relation.

\section{REFERENCES}

1. P. Alexandroff and P. Urysohn, Memoire sur less Espaces Topologiques Compact, Verh. Nederl. Akad. Wetensch. Amsterdam, 14 (1929), 1-96.

2. A. Arhangel'skii, The power of compacta with the first axiom of countability, Dokl. Akad. Nauk. S.S.S.R., 187 (1969), 967-968 (Soviet Math. Dokl., 10 (1969), 951-955).

3. R. Engelking, Outline of General Topology, John Wiley and Sons Inc., New York, 1968.

4. I. Juhasz, Cardinal functions in topology, Mathematical Centre Tracts 34, Mathematisch Centrum, Amsterdam, 1975.

5. M. Katétov, Complete normality and Cartesian products, Fund. Math., 35 (1948), 271-274.

6. R. Pol, Short proofs of two theorems on cardinality of topological spaces, Bull. Acad. Polon. Sci. Ser. Math. Astr. Phys., 22 (1974), 1245-1249.

7. T. Przymusinski and F. D. Tall, The undecidability of the existence of a nonsepara ble normal Moore space satisfying the countable chain condition, Fund. Math., 85 (1974), 291-297.

8. M. E. Rudin, Lectures in set-theoretic topology, C.B.M.S. Regional Conference series in Mathematics No. 23 (1974).

9. M. Ulmer, Products of weakly $\mathbf{\aleph}$-compact spaces, Trans. Amer. Math. Soc., 170 (1972), 279-284.

10. R. G. Woods, Characterizations of some $C^{*}$-embedded subspaces of $\beta N$, Pacific J. Math., 65 (1976), 573-579.

Received March 3, 1978 and in revised form April 19, 1978. The research of the third-named author was partially supported by Grant No. A7592 from the National Research Council of Canada.

The University of Manitoba,

Winnipeg, Manitoba, Canada 



\section{PACIFIC JOURNAL OF MATHEMATICS}

EDITORS

RICHARD ARENS (Managing Editor)

University of California

Los Angeles, California 90024

C. W. Curtis

University of Oregon

Eugene, OR 97403

C. C. MOORE

University of California

Berkeley, CA 94720
J. DUGUNDJI

Department of Mathematics University of Southern Californı Los Angeles, California 90007

R. Finn and J. Milgram Stanford University Stanford, California 94305

\section{ASSOCIATE EDITORS}

E. F. BECKENBACH

B. H. NEUMANN

F. WOLF

K. YoSHIDA

\section{SUPPORTING INSTITUTIONS}

UNIVERSITY OF BRITISH COLUMBIA
CALIFORNIA INSTITUTE OF TECHNOLOGY
UNIVERSITY OF CALIFORNIA
MONTANA STATE UNIVERSITY
UNIVERSITY OF NEVADA, RENO
NEW MEXICO STATE UNIVERSITY
OREGON STATE UNIVERSITY
UNIVERSITY OF OREGON

UNIVERSITY OF SOUTHERNEALIFORNIA STANFORD UNIVERSITY UNIVERSITY OF HAWAII UNIVERSITY OF TOKYO UNIVERSITY OF UTAH WASHINGTON STATE UNIVERSITY UNIVERSITY OF WASHINGTON 


\section{Pacific Journal of Mathematics \\ Vol. 79 , No. 1 \\ May, 1978}

Teófilo Abuabara, A remark on infinitely nuclearly differentiable

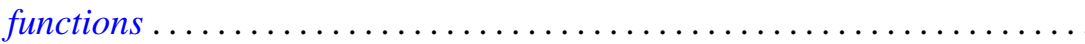

David Fenimore Anderson, Projective modules over subrings of $k[X, Y]$

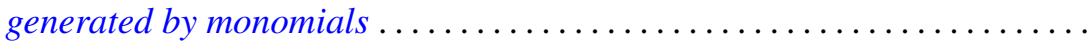

Joseph Barback and Thomas Graham McLaughlin, On the intersection of

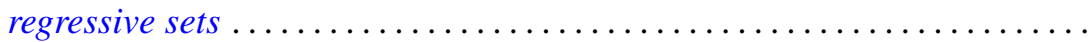

Murray Bell, John Norman Ginsburg and R. Grant Woods, Cardinal inequalities for topological spaces involving the weak Lindelof

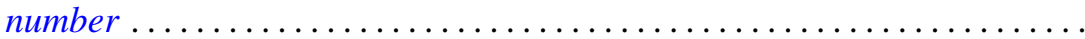

Laurence Richard Boxer, The space of ANRs of a closed surface ............

Zvonko Cerin, Homotopy properties of locally compact spaces at

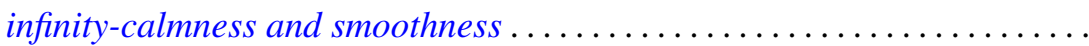

Isidor Fleischer and Ivo G. Rosenberg, The Galois connection between partial functions and relations..................................

John R. Giles, David Allan Gregory and Brailey Sims, Geometrical implications of upper semi-continuity of the duality mapping on a Banach

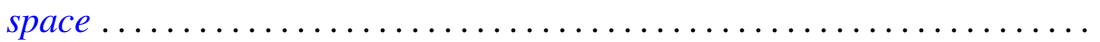

Troy Lee Hicks, Fixed-point theorems in locally convex spaces ............ Hugo Junghenn, Almost periodic functions on semidirect products of transformation semigroups ........................

Victor Kaftal, On the theory of compact operators in von Neumann algebras. II . . . .

Haynes Miller, A spectral sequence for the homology of an infinite

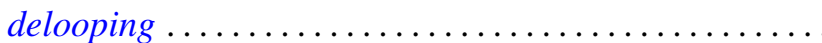

Sanford S. Miller, Petru T. Mocanu and Maxwell O. Reade, Starlike integral operators...

Stanley Stephen Page, Regular FPF rings ...............

Ghan Shyam Pandey, Multipliers for C, 1 summability of Fourier series ...

Shigeo Segawa, Bounded analytic functions on unbounded covering surfaces...

Steven Eugene Shreve, Probability measures and the C-sets of

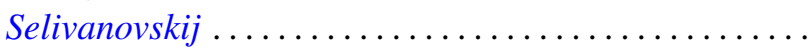

Tor Skjelbred, Combinatorial geometry and actions of compact Lie groups....

Alan Sloan, A note on exponentials of distributions.

Colin Eric Sutherland, Type analysis of the regular representation of a nonunimodular group.

Mark Phillip Thomas, Algebra homomorphisms and the functional

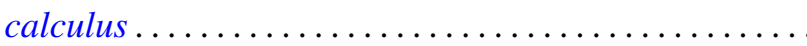

Sergio Eduardo Zarantonello, A representation of $H^{p}$-functions with

$0<p<\infty$. 